\title{
Prevalence and Antimicrobial Resistance of Salmonella serotypes Isolated from Poultry Meat in Japan
}

\author{
Tetsuya Mori, Naoko Okamura, Kanae Kishino, Shintaro Wada, Bizhen Zou, \\ Toyohiko Nanba and Takeshi Ito
}

Incorporated Foundation Tokyo Kenbikyo-in, 5-1, Toyomi-cho, Chuo-ku, Tokyo 104-0055, Japan

\begin{abstract}
The prevalence and antimicrobial susceptibility of Salmonella in 512 poultry meat samples collected from retail stores and poultry-processing plants in Japan between 2015 and 2016 were investigated. The results showed that $55.9 \%$ of poultry meat samples were contaminated with Salmonella, with nine different serotypes represented. The most frequent serovar was Salmonella enterica serovar Infantis, followed by S. Schwarzengrund, together accounting for $78.2 \%$ of the isolates. High antimicrobial resistance rates were observed against tetracycline ( $80.9 \% S$. Infantis and $83.9 \% S$. Schwarzengrund), streptomycin (53.4\% S. Infantis and 76.8\% S. Schwarzengrund), and kanamycin (33.6\% S. Infantis and $82.1 \% S$. Schwarzengrund). All tested isolates were susceptible to colistin and ciprofloxacin. In addition, a high proportion $(65.6 \%$ of $S$. Infantis, $85.7 \%$ of S. Schwarzengrund) of Salmonella isolates were resistant to two or more antimicrobials, and 22 and 17 different resistance patterns were observed in the two strains, respectively. The predominant antibiotic resistance patterns were streptomycintetracycline (32/131, 24.4\% of $S$. Infantis) and streptomycin-kanamycin-tetracycline-sulfamethoxazole/trimethoprim (43/112, $38.4 \%$ of $S$. Schwarzengrund). These data indicate that multidrug-resistant $S$. Infantis and $S$. Schwarzengrund have spread among poultry meat in Japan.
\end{abstract}

Key words: antimicrobial resistance, poultry meat, Salmonella, serotypes

\section{Introduction}

Salmonella is one of the most important foodborne pathogens in Japan. According to the 2015 food poisoning statistics from the Ministry of Health, Labor and Welfare, Salmonella food poisoning was reported in 24 cases and 1918 patients. Infection with Salmonella is commonly acquired by eating raw or undercooked meat, especially poultry. Previous studies have reported the prevalence and antimicrobial susceptibilities of Salmonella in Japan ${ }^{1-7)}$. However, continuous study is important for precise science-based assessment of antimicrobial-resistant bacteria caused by antimicrobial use in food-producing animals, and their risks to human health. The aim of this study was to investigate the current prevalence and antimicrobial susceptibility of Salmonella in poultry meat in Japan.

\section{Materials and Methods}

\section{Sample Collection}

A total of 357 samples of domestic poultry breast meat were collected from retail stores in major cities such as Tokyo, Osaka, Nagoya in Japan between June and September 2015, and 155 samples were collected in eleven poultryprocessing plants in Japan between July 2015 and January 2016. All samples were transported under cold conditions to the laboratory for microbial examination.

\section{Salmonella identification}

Each meat sample (25 g) was homogenized in a stom-

Received: 15 February 2018; Accepted: 2 April 2018; Published online: 31 May 2018

Corresponding author: Tetsuya Mori, Incorporated Foundation Tokyo Kenbikyo-in, 5-1, Toyomi-cho, Chuo-ku, Tokyo 104-0055, Japan (t-mori@kenko-kenbi.or.jp)

The contents of this article reflect solely the view of the author(s). 
Table 1. Distribution of Salmonella serotypes isolated from poultry meat

\begin{tabular}{cccc}
\hline \multirow{2}{*}{ Serotype } & \multicolumn{3}{c}{ Number of isolates (\%) } \\
\cline { 2 - 4 } & Retail & Poultry-processing plant & Total \\
\hline$S$. Infantis & 113 & 18 & $131(42.2)$ \\
$S$. Schwarzengrund & 63 & 49 & $112(36.0)$ \\
$S$. Manhattan & 25 & 29 & $54(17.4)$ \\
$S$. Typhimurium & 4 & 0 & $4(1.3)$ \\
$S$. Agona & 3 & 0 & $3(1.0)$ \\
$S$. Blockley & 2 & 0 & $2(0.6)$ \\
$S$. Anatum & 1 & 0 & $1(0.3)$ \\
$S$. Cubana & 1 & 0 & $1(0.3)$ \\
$S$. Montevideo & 1 & 0 & $1(0.3)$ \\
Untypeable & 1 & 1 & $2(0.6)$ \\
\hline Total & 214 & 97 & $311(100)$ \\
\hline
\end{tabular}

acher with $225 \mathrm{~mL}$ of buffered peptone water (Oxoid Ltd., Hampshire, England) and then incubated at $36^{\circ} \mathrm{C}$ for 22 hours. After incubation, $1.0 \mathrm{~mL}$ was inoculated into $10 \mathrm{~mL}$ of tetrathionate broth (Oxoid) and another $0.1 \mathrm{~mL}$ was into $10 \mathrm{~mL}$ of Rappaport-Vassiliadis broth (Oxoid) and incubated at $42^{\circ} \mathrm{C}$ for 22 hours. The enrichment culture broths were subcultured on deoxycholate hydrogen sulfide lactose agar (Kyokuto Pharmaceutical Industrial Co. Ltd., Tokyo, Japan) and CHROMagar Salmonella agar (Kanto Chemical Co. Ltd., Tokyo, Japan) and incubated at $36^{\circ} \mathrm{C}$ for 22 hours. Presumptive Salmonella isolates were identified using triple sugar iron agar (Nissui Pharmaceutical Co. Ltd. Tokyo, Japan) and lysine indole motility agar (Nissui Pharmaceutical) at $36^{\circ} \mathrm{C}$ for 18 hours. Salmonella isolates were further serotyped by slide agglutination with O-antisera (Denka Seiken Co. Ltd., Tokyo, Japan) and tube agglutination with $\mathrm{H}$-antisera (Denka Seiken). Serovars were determined on the basis of reaction with $\mathrm{O}$ and $\mathrm{H}$ group antigens according to the Kauffman-White method $^{8)}$.

\section{Antimicrobial Susceptibility Testing}

Antimicrobial susceptibility testing was performed according to Clinical and Laboratory Standards Institute method M07-A99). Minimum inhibitory concentrations of 12 antimicrobial agents (ampicillin, cefazolin, cefotaxime, streptomycin, gentamicin, kanamycin, tetracycline, chloramphenicol, colistin, nalidixic acid, ciprofloxacin, and sulfamethoxazole/trimethoprim) were determined using a dry broth microdilution panel. The breakpoints were interpreted according to the Japanese Veterinary Antimicrobial Resistance Monitoring System ${ }^{1)}$.

\section{Results and Discussion}

In this study, Salmonella was isolated from 196 of 357 $(54.9 \%)$ retail poultry meats and 90 of 155 (58.1\%) meats collected at poultry-processing plants, respectively. The overall prevalence of Salmonella in poultry meat was $55.9 \%$ (286/512). The prevalence of Salmonella in retail poultry meat in Japan was reported to be $54.0 \%$ in $2012^{2)}$. Taguchi et al. ${ }^{3)}$ investigated the prevalence of Salmonella in domestic chicken meat samples over 6-years from 2006-2011, and found that the proportion of samples positive for Salmonella spp. gradually increased, from $34.8 \%$ in 2006 to $56.5 \%$ in 2011. The contamination rate of poultry meat in this study is consistent with these reports. However, it is difficult to compare the results with previous reports because of differences in sampling methods, especially the season in which the samples were collected and the isolation methods. A total of 311 Salmonella isolates were obtained from 286 positive samples (Table 1). Among the isolates found in this study, nine different serotypes were completely identified. $S$. Infantis $(131 / 311,42.2 \%)$ was the most frequently detected serotype, followed by $S$. Schwarzengrund (112/311, 36.0\%), $S$. Manhattan (54/311, 17.4\%), S. Typhimurium (4/311, 1.3\%), $S$. Agona (3/311, 1.0\%), $S$. Blockley (2/311, 0.6\%), $S$. Anatum $(1 / 311,0.3 \%), S$. Cubana $(1 / 311,0.3 \%)$, and $S$. Montevideo $(1 / 311,0.3 \%$; Table 1). This is consistent with previous reports demonstrated that $S$. Infantis exhibited the highest prevalence among detected Salmonella serovars in retail poultry meat in Japan, followed by $S$. Schwarzengrund and $S$. Manhattan ${ }^{2,3)}$. Sasaki et al. ${ }^{4)}$ determined the prevalence and serovars of Salmonella isolated from broiler flocks in seven regions of Japan, and found that $S$. Infantis was the 
Table 2. Prevalence and antimicrobial resistance patterns of Salmonella Infantis $(\mathrm{n}=131)$ isolated from poultry meat

\begin{tabular}{|c|c|c|c|c|c|c|c|c|c|c|c|}
\hline \multicolumn{12}{|c|}{$\begin{array}{c}\text { Antimicrobials }{ }^{a} \\
n(\%)\end{array}$} \\
\hline $\mathrm{ABPC}$ & CEZ & CTX & SM & GM & KM & $\mathrm{TC}$ & $\mathrm{CP}$ & CL & NA & CPFX & ST \\
\hline $16(12.2)$ & $11(8.4)$ & $7(5.3)$ & $70(53.4)$ & $0(0)$ & $44(33.6)$ & $106(80.9)$ & $0(0)$ & $0(0)$ & $14(10.7)$ & $0(0)$ & $3(2.3)$ \\
\hline \multicolumn{2}{|c|}{ Nunber of antimicrobials } & \multicolumn{10}{|c|}{ Antimicrobial resistance profile (n) } \\
\hline \multicolumn{2}{|c|}{6} & \multicolumn{10}{|c|}{ ABPC CEZ CTX SM KM TC ( 2 ), ABPC CEZ CTX SM TC NA ( 1 ) } \\
\hline \multicolumn{2}{|c|}{5} & \multicolumn{10}{|c|}{ ABPC CEZ CTX SM TC ( 3 ), ABPC CEZ CTX TC NA ( 1 ), ABPC SM KM TC ST ( 1 ) } \\
\hline \multicolumn{2}{|c|}{4} & \multicolumn{10}{|c|}{ ABPC CEZ SM TC ( 3 ), SM KM TC NA ( 3 ), ABPC CEZ KM TC ( 1 ), ABPC SM KM TC ( 1 ), KM TC NA ST ( 1 ) } \\
\hline \multicolumn{2}{|c|}{3} & \multicolumn{10}{|c|}{ SM KM TC ( 20 ), SM TC NA ( 3 ), ABPC SM ST ( 1 ), KM TC NA ( 1 ) } \\
\hline \multicolumn{2}{|c|}{2} & \multicolumn{10}{|c|}{ SM TC ( 32 ), KM TC ( 8 ), ABPC KM ( 2 ), KM NA ( 1 ), TC NA ( 1 ) } \\
\hline \multirow{2}{*}{\multicolumn{2}{|c|}{1}} & \multicolumn{10}{|c|}{$\mathrm{TC}(24), \mathrm{KM}(3), \mathrm{NA}(2)$} \\
\hline & & \multicolumn{10}{|c|}{ Susceptible ( 16 ) } \\
\hline
\end{tabular}

a ABPC: ampicillin, CEZ: cefazolin, CTX: cefotaxime, SM: streptomycin, KM: kanamycin, TC: tetracycline,

NA: nalidixic acid, ST: sulfamethoxazole/trimethoprim

Table 3. Prevalence and antimicrobial resistance patterns of Salmonella Schwarzengrund $(\mathrm{n}=112)$ isolated from poultry meat

\begin{tabular}{|c|c|c|c|c|c|c|c|c|c|c|c|}
\hline \multicolumn{12}{|c|}{$\begin{array}{c}\text { Antimicrobials }{ }^{a} \\
n(\%)\end{array}$} \\
\hline $\mathrm{ABPC}$ & CEZ & CTX & SM & GM & $\mathrm{KM}$ & $\mathrm{TC}$ & $\mathrm{CP}$ & CL & NA & CPFX & ST \\
\hline $2(1.8)$ & $0(0)$ & $0(0)$ & $86(76.8)$ & $2(1.8)$ & $92(82.1)$ & $94(83.9)$ & $1(0.9)$ & $0(0)$ & $6(5.4)$ & $0(0)$ & $57(50.9)$ \\
\hline \multicolumn{2}{|c|}{ Nunber of antimicrobials } & \multicolumn{10}{|c|}{ Antimicrobial resistance profile (n) } \\
\hline \multicolumn{2}{|c|}{7} & \multicolumn{10}{|c|}{ ABPC SM GM KM TC CP ST ( 1 ) } \\
\hline \multicolumn{2}{|c|}{5} & \multicolumn{10}{|c|}{ ABPC SM KM TC ST ( 1 ), SM KM TC NA ST ( 3 ) } \\
\hline \multicolumn{2}{|c|}{4} & \multicolumn{10}{|c|}{ SM KM TC ST ( 43 ), SM KM TC NA ( 1 ) } \\
\hline \multicolumn{2}{|c|}{3} & \multicolumn{10}{|c|}{ SM KM TC ( 17 ), KM TC ST ( 5 ), SM TC ST ( 4 ), SM GM KM ( 1 ), KM TC NA ( 1 ) } \\
\hline & & \multicolumn{10}{|c|}{$\operatorname{SM~TC~(~} 13$ ), KM TC ( 4 ), SM KM ( 1 ), KM NA ( 1 ) } \\
\hline \multicolumn{2}{|c|}{1} & \multicolumn{10}{|c|}{$\mathrm{KM}(13), \mathrm{SM}(1), \mathrm{TC}(1)$} \\
\hline & & \multicolumn{10}{|c|}{ Susceptible (1) } \\
\hline
\end{tabular}

a ABPC: ampicillin, CEZ: cefazolin, CTX: cefotaxime, SM: streptomycin, KM: kanamycin, TC: tetracycline,

NA: nalidixic acid, ST: sulfamethoxazole/trimethoprim

most frequent serovar isolated in all seven regions, whereas $S$. Manhattan and $S$. Schwarzengrund were frequently found only in western Japan. Thus, the predominant serovars found in broilers is different among regions in Japan; however, the geographical distribution of Salmonella in this study was still unclear.

Antimicrobial susceptibility was determined for 243 isolates (131 S. Infantis and 112 S. Schwarzengrund; Table 2, 3). High antimicrobial resistance rates were observed against tetracycline $(80.9 \% \quad S$. Infantis and $83.9 \% \quad S$. Schwarzengrund), streptomycin $(53.4 \% S$. Infantis and $76.8 \% \mathrm{~S}$.
Schwarzengrund), and kanamycin (33.6\% $S$. Infantis and $82.1 \%$ S. Schwarzengrund). No isolates were identified that were resistant to colistin and ciprofloxacin. Of the 243 isolates, $86(65.6 \%) S$. Infantis and $96(85.7 \%) S$. Schwarzengrund isolates were resistant to two or more antimicrobials, including third-generation cephalosporin (cefotaxime) -resistant Salmonella Infantis (Table 2, 3). The most frequent multidrug resistance pattern among $S$. Infantis isolates was streptomycin-tetracycline (32 isolates), followed by streptomycin-kanamycin-tetracycline (20 isolates; Table 2). The most frequent multidrug resistance pattern among $S$. 
Schwarzengrund isolates was streptomycin-kanamycintetracycline-sulfamethoxazole/trimethoprim (43 isolates), followed by streptomycin-kanamycin-tetracycline (17 isolates; Table 3). Consistently, previous reports demonstrated that Salmonella isolates in poultry meat in Japan were very frequently resistant to tetracycline and streptomycin ${ }^{2,4-7)}$. In addition, the majority of Salmonella isolates in previous studies were resistant to multiple antimicrobials ${ }^{2,4-7)}$. In this study, multidrug resistant strains of $S$. Infantis with resistance to third-generation cephalosporin (cefotaxime) were found. The development of nontyphoidal Salmonella isolates resistant to extended-spectrum cephalosporin represents another substantial public health concern ${ }^{10)}$. In conclusion, these results indicate that multidrug-resistant $S$. Infantis and $S$. Schwarzengrund are widely distributed in domestic poultry meat, and thus these meats may be an important carrier of Salmonella. To ensure precise and current sciencebased assessments of the risks of antimicrobial-resistant bacteria to human health in Japan, continuous study should be performed to investigate Salmonella contamination and antimicrobial resistance.

\section{Acknowledgments}

This work was supported by the Food Safety Commission, Cabinet Office, Government of Japan.

\section{Conflict of interest}

The authors have no conflict of interest.

\section{References}

1. National Veterinary Assay Laboratory Ministry of Agriculture, Forestry and Fisheries. A Report on the Japanese Veterinary Antimicrobial Resistance Monitoring System -2008 to 2011-. 2013. Accessed February 14, 2018.

2. Furukawa I, Ishihara T, Teranishi H, et al. Prevalence and characteristics of Salmonella and Campylobacter in retail poultry meat in Japan. Jpn J Infect Dis. 2017; 70: 239-247. PMID:27580577, doi:10.7883/yoken.JJID.2016.164

3. Taguchi M, Kawahara R, Seto K, Harada T, Kumeda Y. Extended-spectrum $\beta$-lactamase- and AmpC $\beta$-lactamaseproducing Salmonella enterica strains isolated from domestic retail chicken meat from 2006 to 2011. Jpn J Infect Dis. 2012; 65: 555-557. PMID:23183214, doi:10.7883/yoken.65.555

4. Sasaki Y, Ikeda A, Ishikawa K, et al. Prevalence and antimicrobial susceptibility of Salmonella in Japanese broiler flocks. Epidemiol Infect. 2012; 140: 2074-2081. PMID:22281015, doi:10.1017/S0950268812000039

5. Iwabuchi E, Yamamoto S, Endo Y, Ochiai T, Hirai K. Prevalence of Salmonella isolates and antimicrobial resistance patterns in chicken meat throughout Japan. J Food Prot. 2011; 74: 270-273. PMID:21333147, doi:10.4315/0362-028X.JFP$10-215$

6. Asai T, Murakami K, Ozawa M, Koike R, Ishikawa H. Relationships between multidrug-resistant Salmonella enterica Serovar Schwarzengrund and both broiler chickens and retail chicken meats in Japan. Jpn J Infect Dis. 2009; 62: 198-200. PMID:19468180

7. Ono K. Quantitative contamination level of Campylobacter and Salmonella species in commercial chicken meat and their drug susceptibility. J Jpn Vet Med Assoc. 2014; 67: 442-448. Japanese. doi:10.12935/jvma.67.442

8. Grimont PAD, Weill F. Antigenic formulae of the Salmonella serovars, 9th edition. WHO Collaborating Center for Reference and Research on Salmonella, Paris, France: Institut Pasteur; 2007.

9. Clinical and Laboratory Standards Institute Methods for dilution antimicrobial susceptibility tests for bacteria that grow aerobically; Approved Standard, 9th ed. M07-A9, Wayne, USA: Clinical and Laboratory Standards Institute; 2012.

10. Crump JA, Sjölund-Karlsson M, Gordon MA, Parry CM. Epidemiology, Clinical Presentation, Laboratory Diagnosis, Antimicrobial Resistance, and Antimicrobial Management of Invasive Salmonella Infections. Clin Microbiol Rev. 2015; 28: 901-937. PMID:26180063, doi:10.1128/CMR.00002-15 